\title{
New Startup Method Using Internal Momentum Management of Variable-Speed Control Moment Gyroscopes
}

\author{
Dohee Kim* \\ University of Florida, Gainesville, Florida 32611 \\ Frederick A. Leve $₫$ \\ U.S. Air Force Research Laboratory, Albuquerque, New Mexico 87117 \\ and \\ Norman G. Fitz-Coy” and Warren E. Dixon \\ University of Florida, Gainesville, Florida 32611
}

\begin{abstract}
DOI: $\underline{10.2514 / 1.55984}$
An initial startup method for a spacecraft actuated by a pyramidal arrangement of variable-speed control moment gyroscopes is developed. The method provides closed-loop internal momentum tracking control to enable the flywheels to start from rest and reach desired wheel speeds. The proposed controller functioning as a variable-speed control moment gyroscope steering law is developed in terms of the gimbal rates and the flywheel accelerations, which are weighted by a singularity measure. Specifically, using null motion, a strategy is developed to simultaneously perform gimbal reconfiguration for singularity avoidance and internal momentum management for flywheel startup. A Lyapunov-based stability analysis is used to prove asymptotic attitude tracking and exponential internal momentum tracking despite the effects of uncertain, time-varying satellite inertia properties and uncertain actuator inertia properties. Numerical simulations illustrate the performance of the adaptive controller as a variablespeed control moment gyroscope steering law.
\end{abstract}

\begin{tabular}{|c|c|c|}
\hline \multicolumn{3}{|r|}{ Nomenclature } \\
\hline$\hat{a}_{G_{i}}, \hat{a}_{W_{i}}, \hat{a}_{T_{i}}$ & $=$ & $\begin{array}{l}\text { gimbal axis, spinning wheel axis, and transverse } \\
\text { axis }\end{array}$ \\
\hline $\mathcal{B}$ & $=$ & control moment gyroscope-fixed basis \\
\hline$C_{G}, C_{W}, C_{T}$ & $=$ & $\begin{array}{l}\text { Jacobian matrix of gimbal axis, spinning wheel } \\
\text { axis, and transverse axis }\end{array}$ \\
\hline$e, r, \mu$ & $=$ & $\begin{array}{l}\text { quaternion tracking error, filter tracking error, } \\
\text { angular momentum tracking error }\end{array}$ \\
\hline $\mathcal{F}_{S}, \mathcal{F}_{S_{d}}$ & $=$ & satellite and desired satellite body-fixed frames \\
\hline$f \quad v_{d}$ & $=$ & objective function measuring singularity \\
\hline$g$ & $=$ & $\begin{array}{l}\text { control signal to track desired flywheel angular } \\
\text { momentum }\end{array}$ \\
\hline$g_{E}$ & $=$ & external torque applied to satellite \\
\hline$H$ & $=$ & total angular momentum \\
\hline$h, h_{d}$ & & $\begin{array}{l}\text { flywheel angular momentum vector, desired } \\
\text { flywheel angular momentum vector }\end{array}$ \\
\hline$k, k_{m}, k_{\gamma}, k_{w}$ & $=$ & positive constant gains \\
\hline & $=$ & inertial reference frame \\
\hline$I_{G}^{\mathrm{CMG}}, I_{W}$ & $=$ & gimbal inertia matrix, flywheel inertia matrix \\
\hline$J$ & $=$ & total satellite inertia matrix \\
\hline$N_{1}, N_{2}$ & $=$ & null solutions of control input $\dot{\eta}(t)$ \\
\hline & $=$ & unit quaternion \\
\hline$R, R_{d}, \tilde{R}$ & $=$ & $\begin{array}{l}\text { actual rotation matrix, desired rotation matrix, } \\
\text { and attitude tracking error matrix }\end{array}$ \\
\hline$R_{1}, R_{2}$ & $=$ & range solutions of control input $\dot{\eta}(t)$ \\
\hline$S, W$ & $=$ & null-motion weight matrix, mode weight matrix \\
\hline$Y_{1}, Y_{2}$ & $=$ & regression matrices \\
\hline$\alpha$ & $=$ & a positive constant \\
\hline
\end{tabular}

Received 18 August 2011; revision received 19 January 2012; accepted for publication 20 February 2012. Copyright $@ 2012$ by the American Institute of Aeronautics and Astronautics, Inc. All rights reserved. Copies of this paper may be made for personal or internal use, on condition that the copier pay the $\$ 10.00$ per-copy fee to the Copyright Clearance Center, Inc., 222 Rosewood Drive, Danvers, MA 01923; include the code 0731-5090/12 and \$10.00 in correspondence with the CCC.

${ }^{*}$ Graduate Student, Department of Mechanical and Aerospace Engineering.

${ }^{\dagger}$ Research Aerospace Engineer, Space Vehicles Directorate.

¥Associate Professor, Department of Mechanical and Aerospace Engineering.

\begin{tabular}{|c|c|}
\hline $\begin{array}{l}\gamma, \sigma \\
\delta, \ddot{\delta}, \ddot{\delta}\end{array}$ & $\begin{array}{l}=\text { singularity measure index, null motion } \\
=\text { gimbal angle, gimbal rate, and gimbal } \\
\text { acceleration vector }\end{array}$ \\
\hline$\dot{\eta}$ & $\begin{aligned}= & \text { composite control input consisting of flywheel } \\
& \text { acceleration and gimbal rate }\end{aligned}$ \\
\hline$\theta_{1}, \theta_{2}$ & unknown constants \\
\hline$\Omega, \dot{\Omega}, \Omega_{d}$ & $\begin{aligned}= & \text { flywheel speed, flywheel acceleration, and } \\
& \text { desired flywheel speed vector }\end{aligned}$ \\
\hline$\omega, \omega_{d}, \tilde{\omega}$ & $\begin{array}{l}\text { satellite angular velocity vector, desired satellite } \\
\text { angular velocity vector, satellite angular } \\
\text { velocity error }\end{array}$ \\
\hline & satellite angular velocity projected to $\mathcal{B}$ \\
\hline
\end{tabular}

\section{Introduction}

$\mathbf{V}$ ARIOUS spacecraft momentum control devices such as momentum wheels (MWs), reaction wheels (RWs), and control moment gyroscopes (CMGs) are used to maintain and/or perform precise attitude maneuvers. For spacecraft hosting these devices, the operational spin rate of the wheel must be initially spun up and obtained.

Several wheel initialization methods have been investigated for spin up of MWs and RWs [1-3]. A pitch MW method can be used during an initial attitude acquisition mode, where the wheel requires magnetorquers to maintain its spin rate, while providing attitude stabilization [1]. Such a method can also be used to acquire the gyroscopic stiffness along the roll and yaw axes [2]. The attitude determination and control system uses a sequential mode change of a MW and the magnetorquers, during spin up, while maintaining a gravity-gradient stabilized attitude profile. Wheel spin up and attitude stabilization has also been shown when transitioning from safe hold mode to initial attitude acquisition mode by using four RWs with magnetorquers [3].

Various large space structures such as Skylab, Mir, and the International Space Station have employed CMGs to take advantage of their torque amplification and power saving properties when compared with RWs. Despite CMGs performance benefits, singularities inherent in their use make the control of them complex [4-9]. Various solutions address the CMG singularity issue [10-13] and, with the development of mini-CMGs $[\underline{8}, \underline{14}-\underline{23}]$, several recent 
space missions using CMGs have been launched or scheduled [2426]. Previous space missions using CMGs have used a separate feedback control loop to spin up the rotors to the required spin rate and maintain it, while securing attitude stabilization using additional devices such as magnetorquers $[25,27]$. However, use of CMGs with magnetorquers requires very long spin-up times due to the power constraints of CMGs (i.e., shaft power constraints) and the less than desirable responsiveness of magnetorquers in providing torque or dumping large amounts of angular momentum than may be collected from external disturbance torques during the spin-up time. In addition, many zero-momentum configurations of CMG arrays are singular (i.e., rooftop arrangements) [10] and thus spin up in many cases is done open loop.

Variable-speed CMGs (VSCMGs) combine properties of RWs and CMGs, in which the spinning rotor is accelerated and gimbaled, providing an extra controllable degree of freedom $[4,28,29]$. The extra degree of freedom present in VSCMGs provides an avenue to condense the initial startup and initial attitude acquisition mode into one step, while dumping the angular momentum from external disturbance torques on-the-fly with magnetorquers. Several flywheel speed regularization approaches appear in the literature $[5,30,31]$. For example, VSCMGs null motion can be used to achieve each preferred gimbal angle and wheel speed sets [30]. However, the preferred sets need to be calculated offline. An example in [31] of an online technique for wheel speed equalization is a local gradient method that obtains consistent performance for a VSCMGs-based integrated power and attitude control system.

An adaptive controller-based VSCMG steering law developed in this paper exploits internal momentum management (i.e., the management of flywheel speeds) and singularity avoidance (i.e., singularities associated with CMGs not VSCMGs), while simultaneously yielding precise attitude control. The closed-loop VSCMG steering law provided from the adaptive controller yields simultaneous asymptotic attitude tracking, exponential internal momentum tracking, and singularity avoidance, without a separate feedback control loop. Control moment gyroscope singularities that are hyperbolic (i.e., assuming nondegenerate hyperbolic) are avoided solely through null motion such that the amount of VSCMG flywheel accelerations is minimal and used entirely for internal momentum management and elliptic singularity avoidance. The VSCMG steering law is developed for a satellite with an uncertain, state-dependent inertia along with an uncertain inertia in the VSCMG actuators.

The paper is organized as follows. Sections II and III develop the kinematic and dynamic models of the system. The overall control objectives are described in Sec. IV and a detailed analysis of the adaptive VSCMGs steering law is presented in Sec. V. Simulation results in Sec. VI illustrate the performance of the proposed adaptive controller as a VSCMGs steering law. Conclusions are presented in Sec. VII.

\section{Kinematic Model}

The rotational kinematics of a rigid-body satellite can be expressed as [르]

$$
\begin{gathered}
\dot{q}_{v}=\frac{1}{2}\left(q_{v}^{\times} \omega+q_{0} \omega\right) \\
\dot{q}_{0}=-\frac{1}{2} q_{v}^{T} \omega
\end{gathered}
$$

where $\omega(t) \in \mathbb{R}^{3}$ denotes the satellite angular velocity, and $q(t) \triangleq$ $\left\{q_{0}(t), q_{v}(t)\right\} \in \mathbb{R} \times \mathbb{R}^{3}$ represents the unit quaternion describing the orientation of the satellite body-fixed frame $\mathcal{F}_{S}$ with respect to the inertial reference frame $\mathcal{I}$, subject to the constraint

$$
q_{v}^{T} q_{v}+q_{0}^{2}=1
$$

In Eq. (1), $q_{v}^{\times} \forall q_{v}=\left[q_{v_{1}}, q_{v_{2}}, q_{v_{3}}\right]^{T}$ denotes the following skewsymmetric matrix:

$$
q_{v}^{\times}=\left[\begin{array}{ccc}
0 & -q_{v_{3}} & q_{v_{2}} \\
q_{v_{3}} & 0 & -q_{v_{1}} \\
-q_{v_{2}} & q_{v_{1}} & 0
\end{array}\right]
$$

Rotation matrices that bring $\mathcal{I}$ onto $\mathcal{F}_{S}$ and $\mathcal{I}$ onto the desired body-fixed orientation $\mathcal{F}_{S_{d}}$ are denoted by $R\left(q_{0}, q_{v}\right) \in S O(3)$ and $R_{d}\left(q_{0 d}, q_{v d}\right) \in S O(3)$, respectively, and are defined as

$$
\begin{gathered}
R \triangleq\left(q_{0}^{2}-q_{v}^{T} q v\right) I_{3}+2 q_{v} q_{v}^{T}-2 q_{0} q_{v}^{\times} \\
R_{d} \triangleq\left(q_{0 d}^{2}-q_{v d}^{T} q_{v d}\right) I_{3}+2 q_{v d} q_{v d}^{T}-2 q_{0 d} q_{v d}^{\times}
\end{gathered}
$$

where $I_{3}$ denotes the $3 \times 3$ identity matrix, and $q_{d}(t) \triangleq$ $\left\{q_{0 d}(t), q_{v d}(t)\right\} \in \mathbb{R} \times \mathbb{R}^{3}$ represents the desired unit quaternion that describes the orientation of $\mathcal{F}_{S_{d}}$ with respect to $\mathcal{I}$.

\section{Dynamic Model}

The total angular momentum $H(\omega, \dot{\delta}, \Omega) \in \mathbb{R}^{3}$ of a rigid VSCMGactuated satellite can be written as [27]

$$
H=J \omega+C_{G}\left[I_{G}^{\mathrm{CMG}}\right]^{d} \dot{\delta}+C_{W}\left[I_{W}\right]^{d} \Omega
$$

where the angular momentum of the VSCMG is expressed in terms

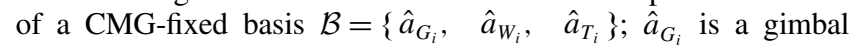
axis, $\hat{a}_{W_{i}}$ is a spinning wheel axis, and $\hat{a}_{T_{i}}$ is a transverse axis of $i$ th CMG unit, as shown in Fig. $1 ; C_{G}, C_{W}(\delta), C_{T}(\delta) \in \mathbb{R}^{3 \times 4}$ are defined as $C_{G} \triangleq\left[\begin{array}{llll}\hat{a}_{G_{1}} & \hat{a}_{G_{2}} & \hat{a}_{G_{3}} & \hat{a}_{G_{4}}\end{array}\right], C_{W} \triangleq\left[\begin{array}{llll}\hat{a}_{W_{1}} & \hat{a}_{W_{2}} & \hat{a}_{W_{3}} & \hat{a}_{W_{4}}\end{array}\right]$, $C_{T} \triangleq\left[\begin{array}{llll}\hat{a}_{T_{1}} & \hat{a}_{T_{2}} & \hat{a}_{T_{3}} & \hat{a}_{T_{4}}\end{array}\right]$, and the inertia matrices $\left[I_{G}^{\mathrm{CMG}}\right]^{d}$, $\left[I_{W}\right]^{d} \in \mathbb{R}^{4 \times 4}$ are $\left[I_{G}^{\mathrm{CMG}}\right]^{d} \triangleq \operatorname{diag}\left(\left[\begin{array}{llll}I_{G}^{\mathrm{CMG}_{1}} & I_{G}^{\mathrm{CMG}_{2}} & I_{G}^{\mathrm{CMG}_{3}} & I_{G}^{\mathrm{CMG}_{4}}\end{array}\right]\right)$, $\left[I_{W}\right]^{d} \triangleq \operatorname{diag}\left(\left[\begin{array}{llll}I_{W_{1}} & I_{W_{2}} & I_{W_{3}} & I_{W_{4}}\end{array}\right]\right)$, where $\dot{\delta}(t), \Omega(t) \in \mathbb{R}^{4}$ are the gimbal rate and flywheel speed vector, respectively; and $\left[I_{G}^{\mathrm{CMG}}\right]^{d}$ is the unknown constant positive-definite, symmetric about its gimbal axis, gimbal inertia matrix, and $\left[I_{W}\right]^{d}$ is the known constant positivedefinite, symmetric about its spin axis, flywheel inertia matrix. Because a structure for flywheel is quite simple and symmetric compared with a gimbal including complex structures and antisymmetry, there is little variation for uncertainty of flywheel inertia, whereas gimbal inertia properties have more parametric uncertainty variations. Hence, considering flywheel inertia and gimbal inertia to be known and unknown, respectively, is reasonable. In Eq. (6), the uncertain total satellite inertia matrix $J(\delta) \in \mathbb{R}^{3 \times 3}$ is positive definite and symmetric such that

$$
\frac{1}{2} \lambda_{\min }\{J\}\|\xi\|^{2} \leq \xi^{T} J \xi \leq \frac{1}{2} \lambda_{\max }\{J\}\|\xi\|^{2} \quad \forall \xi \in \mathbb{R}^{3}
$$

where $\lambda_{\min }\{J\}, \lambda_{\max }\{J\} \in \mathbb{R}$ are the minimum and maximum principal inertias of $J(\delta)$, and $\delta(t) \in \mathbb{R}^{4}$ is the gimbal angle. The equation of motion for a rigid VSCMG-actuated satellite can be written as

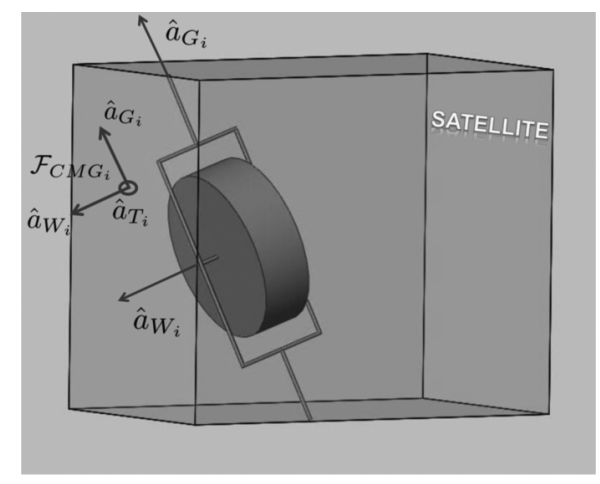

Fig. 1 Geometry of satellite with $i$ th $\operatorname{VSCMG}\left(\mathcal{F}_{\mathrm{CMG}_{i}}\right.$ : $i$ th CMG-fixed frame). 


$$
\begin{aligned}
& g_{E}=\dot{J} \omega+J \dot{\omega}+\omega \times J \omega+C_{G}\left(\left[I_{G}^{\mathrm{CMG}}\right]^{d} \ddot{\delta}-\left[I_{W}\right]^{d}\left[\omega_{T}\right]^{d} \Omega\right) \\
& \quad+C_{W}\left(\left[I_{W}\right]^{d} \dot{\Omega}+\left[I_{G}^{\mathrm{CMG}}\right]^{d}\left[\omega_{T}\right]^{d} \dot{\delta}\right)+C_{T}\left(\left[I_{W}\right]^{d}[\Omega]^{d} \dot{\delta}\right. \\
& \left.\quad+\left[I_{W}\right]^{d}\left[\omega_{G}\right]^{d} \Omega-\left[I_{G}^{\mathrm{CMG}}\right]^{d}\left[\omega_{W}\right]^{d} \dot{\delta}\right)
\end{aligned}
$$

where $g_{E}(\omega, \dot{\omega}, \delta, \dot{\delta}, \ddot{\delta}, \Omega, \dot{\Omega}) \in \mathbb{R}^{3}$ is the external torque applied to the satellite, and $\ddot{\delta}(t), \dot{\Omega}(t) \in \mathbb{R}^{4}$ are the gimbal acceleration and flywheel acceleration. In Eq. (7), the angular velocity projected to $\mathcal{B}$ is denoted as $\left[\omega_{\circ}(t)\right]^{d} \triangleq \operatorname{diag}\left(\left[\begin{array}{llll}\omega_{\circ_{1}}(t) & \omega_{\circ_{2}}(t) & \omega_{\circ_{3}}(t) & \omega_{\circ_{4}}(t)\end{array}\right]\right)$ where $(\circ: G, W, T) \in \mathbb{R}^{4 \times 4}$, and $[\Omega(t)]^{d}$ and $[\dot{\delta}(t)]^{d}$ denote diagonal matrices composed of the vector elements of measurable $\Omega(t)$, $\dot{\delta}(t) \in \mathbb{R}^{4}$, respectively.

\section{Control Objective}

\section{A. Attitude Control Objective}

The attitude control objective is to develop a flywheel acceleration and gimbal rate control law to enable the attitude of $\mathcal{F}_{S}$ to track the attitude of $\mathcal{F}_{S_{d}}$. To quantify the objective, an attitude tracking error denoted by $\tilde{R}\left(e_{0}, e_{v}\right) \in \mathbb{R}^{3 \times 3}$ is defined that brings $\mathcal{F}_{S_{d}}$ onto $\mathcal{F}_{S}$ as

$$
\tilde{R} \triangleq R R_{d}^{T}=\left(e_{0}^{2}-e_{v}^{T} e_{v}\right) I_{3}+2 e_{v} e_{v}^{T}-2 e_{0} e_{v}^{\times}
$$

where the quaternion tracking error $e(t) \triangleq\left\{e_{0}(t), e_{v}(t)\right\} \in \mathbb{R} \times \mathbb{R}^{3}$ is defined as

$$
e_{0} \triangleq q_{0} q_{0 d}+q_{v}^{T} q_{v d} \quad e_{v} \triangleq q_{0 d} q_{v}-q_{0} q_{v d}+q_{v}^{\times} q_{v d}
$$

From the definitions of the quaternion tracking error variables, the following constraint exists:

$$
e_{v}^{T} e_{v}+e_{0}^{2}=1
$$

where

$$
0 \leq\left\|e_{v}(t)\right\| \leq 1 \quad 0 \leq\left|e_{0}(t)\right| \leq 1
$$

where $\|\cdot\|$ represents the standard Euclidean norm. From Eq. (10),

$$
\left\|e_{v}(t)\right\| \rightarrow 0 \Rightarrow\left|e_{0}(t)\right| \rightarrow 1
$$

and, hence, Eq. (ㅇ) can be used to conclude that, if Eq. (12) is satisfied, then the control objective in Eq. (14) is achieved. The openloop quaternion tracking error can be derived by tracking the time derivative of Eq. ()ㅡㄹ as

$$
\dot{e}_{v}=\frac{1}{2}\left(e_{v}^{\times}+e_{0} I\right) \tilde{\omega} \quad \dot{e}_{0}=-\frac{1}{2} e_{v}^{T} \tilde{\omega}
$$

Based on Eq. ( 1 ), the attitude control objective can be stated as

$$
\tilde{R}\left(e_{0}(t), e_{v}(t)\right) \rightarrow I_{3} \quad \text { as } t \rightarrow \infty
$$

The angular velocity of $\mathcal{F}_{S}$ with respect to $\mathcal{F}_{S_{d}}$ expressed in $\mathcal{F}_{S}$, denoted by $\tilde{\omega}(t) \in \mathbb{R}^{3}$, is defined as

$$
\tilde{\omega} \triangleq \omega-\tilde{R} \omega_{d}
$$

where $\omega_{d}(t) \in \mathbb{R}^{3}$ denotes the desired angular velocity of the satellite.

\section{B. Flywheel Angular Momentum Management Objective}

The angular momentum $h(\Omega) \in \mathbb{R}^{4}$ generated by the flywheels of the four VSCMGs can be expressed as

$$
h=\left[I_{W}\right]^{d} \Omega
$$

The flywheel angular momentum management objective is to develop an internal momentum tracking control law resulting from the null solution of the flywheel control input so that the actual angular momentum tracks a desired constant angular momentum $h_{d} \in \mathbb{R}^{4}$, while simultaneously tracking a desired time-varying attitude. To quantify the momentum management objective, an angular momentum tracking error $\mu(\Omega) \in \mathbb{R}^{4}$ is defined as

$$
\mu \triangleq h_{d}-h
$$

where the desired angular momentum is defined as $h_{d} \triangleq\left[I_{W}\right]^{d} \Omega_{d}$.

\section{Controller Development}

\section{A. Adaptive Controller-Based Steering Law Development}

To facilitate the control design, an auxiliary signal $r\left(\omega, e_{0}, e_{v}\right) \in$ $\mathbb{R}^{3}$ is defined as [33]

$$
r \triangleq \omega-\tilde{R} \omega_{d}+\alpha e_{v}
$$

where $\alpha \in \mathbb{R}$ is a positive constant. Using Eqs. (15) and (18), the angular velocity tracking error can be expressed as

$$
\tilde{\omega}=r-\alpha e_{v}
$$

Taking the time derivative of Eq. (18) and multiplying both sides of the resulting expression by $J(\delta)$ yields

$$
J \dot{r}=J \dot{\omega}+J \omega^{\times} \tilde{R} \omega_{d}-J \tilde{R} \dot{\omega}_{d}+\frac{1}{2} J \alpha\left(e_{v}^{\times}+e_{0} I_{3}\right) \tilde{\omega}
$$

where the fact that

$$
\dot{\tilde{R}}=-\omega^{\times} \tilde{R}
$$

was used. After substituting Eq. (7) into Eq. (20), the open-loop error dynamics for $r\left(\omega, e_{0}, e_{v}\right)$ can be written as

$$
J \dot{r}=-Q \dot{\eta}+Y_{1} \theta_{1}-\frac{1}{2} \dot{J} r+C_{G}\left[I_{W}\right]^{d}\left[\omega_{T}\right]^{d} \Omega-C_{T}\left[I_{W}\right]^{d}\left[\omega_{G}\right]^{d} \Omega
$$

under the standard assumption that the gimbal acceleration term $C_{G}\left[I_{G}^{\mathrm{CMG}}\right]^{d} \ddot{\delta}(t)$ is negligible $[\underline{4}, \underline{28}, \underline{34}]$. In Eq. $(\underline{21})$, $Q\left(e_{0}, e_{v}, r, \omega, \delta, \Omega\right) \in \mathbb{R}^{3 \times 8}$ is defined as

$$
Q \triangleq\left[\begin{array}{ll}
C_{W}\left[I_{W}\right]^{d} & C_{W}\left[I_{G}^{\mathrm{CMG}}\right]^{d}\left[\omega_{T}\right]^{d}+C_{T}\left(\left[I_{W}\right]^{d}[\Omega]^{d}\right. \\
\left.-\left[I_{G}^{\mathrm{CMG}}\right]^{d}\left[\omega_{W}\right]^{d}\right)+\frac{\partial J}{\partial \delta}\left(\frac{1}{2} r+\tilde{R} \omega_{d}-\alpha e_{v}\right)
\end{array}\right]
$$

$\dot{\eta}=\left[\begin{array}{ll}\dot{\Omega}^{T} & \dot{\delta}^{T}\end{array}\right]^{T} \in \mathbb{R}^{8 \times 1}$ is a composite control input consisting of the flywheel accelerations and the gimbal rates, and $Y_{1}\left(e_{0}, e_{v}, r, \omega, \delta\right) \theta_{1}$ represents linearly parameterizable uncertainty in terms of a measurable regression matrix $Y_{1}\left(e_{0}, e_{v}, r, \omega, \delta\right) \in \mathbb{R}^{3 \times 10}$ and a vector of 10 unknown constants $\theta_{1} \in \mathbb{R}^{10}$ defined as

$$
Y_{1} \theta_{1} \triangleq-\omega \times J \omega+J \omega^{\times} \tilde{R} \omega_{d}-J \tilde{R} \dot{\omega}_{d}+\frac{1}{2} J \alpha\left(e_{v}^{\times}+e_{0} I_{3}\right) \tilde{\omega}
$$

To compensate for the linearly parameterizable uncertainty present in $Q\left(e_{0}, e_{v}, r, \omega, \delta, \Omega\right)$, another regression matrix denoted by $Y_{2}\left(e_{0}, e_{v}, r, \omega, \delta, \Omega, \dot{\delta}, \dot{\Omega}\right) \in \mathbb{R}^{3 \times 7}$ and a vector of seven unknown constants $\theta_{2} \in \mathbb{R}^{7}$ are defined as

$$
Y_{2} \theta_{2} \triangleq-Q \dot{\eta}
$$

To address the fact that the control input $\dot{\eta}(t)$ is premultiplied by a nonsquare, state-dependent uncertain matrix, an estimate of the uncertainty in Eq. (23), $\hat{Q}(t) \in \mathbb{R}^{3 \times 8}$ is defined as

$$
Y_{2} \hat{\theta}_{2} \triangleq-\hat{Q} \dot{\eta}
$$

where $\hat{\theta}_{2}(t) \in \mathbb{R}^{7}$ is a subsequently designed estimate. Based on Eqs. (23) and (24), the expression in Eq. (21) can be written as

$$
\begin{aligned}
J \dot{r} & =Y_{2} \tilde{\theta}_{2}-\hat{Q} \dot{\eta}+Y_{1} \theta_{1}-\frac{1}{2} \dot{J} r+C_{G}\left[I_{W}\right]^{d}\left[\omega_{T}\right]^{d} \Omega \\
& -C_{T}\left[I_{W}\right]^{d}\left[\omega_{G}\right]^{d} \Omega
\end{aligned}
$$


where the notation $\tilde{\theta}_{2}(t) \in \mathbb{R}^{7}$ is defined as

$$
\tilde{\theta}_{2} \triangleq \theta_{2}-\hat{\theta}_{2}
$$

Based on the open-loop error dynamics of Eq. (25), the composite weighted VSCMG steering law is designed as

$$
\begin{aligned}
\dot{\eta} & =\hat{Q}_{w}^{+}\left(Y_{1} \hat{\theta}_{1}+k r+e_{v}+C_{G}\left[I_{W}\right]^{d}\left[\omega_{T}\right]^{d} \Omega-C_{T}\left[I_{W}\right]^{d}\left[\omega_{G}\right]^{d} \Omega\right) \\
& +\left(I_{8}-\hat{Q}_{w}^{+} \hat{Q}\right) S \sigma
\end{aligned}
$$

where $k \in \mathbb{R}$ denotes a positive control gain, $\hat{Q}_{w}^{+}(\delta, t)=$ $W(\delta) \hat{Q}^{T}(t)\left(\hat{Q}(t) W(\delta) \hat{Q}^{T}(t)\right)^{-1}$, and $W(\delta) \in \mathbb{R}^{8 \times 8}$ denotes a weight matrix that determines if the VSCMGs system uses a CMG mode or an RW mode. Specifically, $W(\delta)$ is designed as [28-30]

$$
W \triangleq\left[\begin{array}{cc}
W_{\Omega} I_{4 \times 4} & 0_{4 \times 4} \\
0_{4 \times 4} & W_{\delta} I_{4 \times 4}
\end{array}\right]
$$

where $W_{\Omega}(\delta) \in \mathbb{R}$ is defined as

$$
W_{\Omega} \triangleq W_{\Omega 0} \exp \left(\lambda_{1} f\right)
$$

$\lambda_{1}, W_{\Omega 0}, W_{\delta} \in \mathbb{R}$ are positive constants, and the objective function $f(\delta)$ measuring the singularity is defined as

$$
f \triangleq-\operatorname{det}\left(C_{T} C_{T}^{T}\right)
$$

The control input in Eq. (27) has an extra degree of freedom resulting from the variable-speed flywheel, and thus enables the VSCMG system to escape a gimbal lock singularity [28]. Because the control input in Eq. (27) is produced by VSCMG units, Eq. (27) is also a solution to an underdetermined system containing flywheel accelerations and gimbal rates as unknowns $[\underline{5}, \underline{28}, \underline{31}]$. The term $\left(I_{8}-\hat{Q}_{w}^{+}(\delta, t) \hat{Q}(t)\right) S(\delta) \sigma(t)$ in Eq. (27) generates the VSCMG null motion for internal momentum management and singularity avoidance. Because the matrices $\hat{Q}_{w}^{+}(\delta, t)$ and $\hat{Q}(t)$ are nonsquare, the pseudoinverse $\hat{Q}_{w}^{+}(\delta, t) \in \mathbb{R}^{8 \times 3}$ is defined so that $\hat{Q}(t) \hat{Q}_{w}^{+}(\delta, t)=I_{3}$, and the matrix $I_{8}-\hat{Q}_{w}^{+}(\delta, t) \hat{Q}(t)$, which projects vectors onto the null space of $\hat{Q}(t)$, satisfies the properties

$$
\begin{gathered}
\left(I_{8}-\hat{Q}_{w}^{+} \hat{Q}\right)\left(I_{8}-\hat{Q}_{w}^{+} \hat{Q}\right)=I_{8}-\hat{Q}_{w}^{+} \hat{Q} \\
\hat{Q}\left(I_{8}-\hat{Q}_{w}^{+} \hat{Q}\right)=0
\end{gathered}
$$

To generate null motion for internal momentum tracking and gimbal reconfiguration, the null-motion $\sigma(t) \in \mathbb{R}^{8}$ is defined as

$$
\sigma \triangleq\left[\begin{array}{ll}
k_{w} g^{T} & k_{\gamma} \frac{\partial \gamma^{T}}{\partial \delta}
\end{array}\right]^{T}
$$

where $k_{w}, k_{\gamma} \in \mathbb{R}$ denote positive constants, $g(t) \in \mathbb{R}^{4}$ is a subsequently designed auxiliary control signal to track the desired flywheel angular momentum, and the second row allows the null motion to perform the gimbal reconfiguration corresponding to a variation of the singularity measure index $\gamma$, which is defined as [11]

$$
\gamma=\gamma_{0} \exp \left(\lambda_{2} f\right)
$$

where the objective function $f$ is defined in Eq. (29), and $\lambda_{2}, \gamma_{0} \in \mathbb{R}$ denote positive constants. The matrix $S(\delta) \in \overline{\mathbb{R}^{8 \times 8}}$ in Eq. (27) is used as a null-motion weight for the VSCMG null motion, and is designed as

$$
S \triangleq \operatorname{diag}\left(\left[s_{w}, s_{g}\right]\right)=\left[\begin{array}{cc}
\operatorname{sech}\left(\frac{1}{k_{d} \operatorname{det}\left(C_{T} C_{T}^{T}\right)+\varepsilon}\right) & 0_{4 \times 4} \\
0_{4 \times 4} & \operatorname{sech}\left(k_{g} \operatorname{det}\left(C_{T} C_{T}^{T}\right)\right)
\end{array}\right]
$$

where $k_{d}, k_{g}, \varepsilon \in \mathbb{R}$. In Eq. $(\underline{33}), s_{w}(\delta), s_{g}(\delta) \in \mathbb{R}^{4 \times 4}$ selectively arbitrate between internal momentum tracking and gimbal reconfiguration, corresponding to how approximate or far the CMG configuration is to a singularity. Gimbal reconfiguration results from null motion when the CMG Jacobian becomes singular (i.e., a hyperbolic singularity), allowing simultaneous attitude and internal momentum tracking with the benefits of torque amplification in CMG mode. When the CMG experiences a degenerate singularity (i.e., even though the null motions exist, the singularity cannot be escaped by the null motions) or when a gimbal lock singularity occurs or no null motion exists (i.e., an elliptic singularity) $[5,7,14,29,30,35]$, then $s_{w}(\delta) \approx 0$, which inhibits momentum tracking so that the VSCMG will operate in RW mode.

The control input $\dot{\eta}(t)$ in Eq. (27) can be partitioned as

$$
\dot{\eta}=\left[\begin{array}{c}
\dot{\Omega} \\
\dot{\delta}
\end{array}\right]=\left[\begin{array}{l}
R_{1}+k_{w} N_{1} \bar{s}_{w} g \\
R_{2}+k_{\gamma} N_{2} \bar{s}_{g} \frac{\partial \gamma}{\partial \delta}
\end{array}\right]
$$

where $\quad R_{i}=\left[\hat{Q}_{w}^{+}\left(Y_{1} \hat{\theta}_{1}+k r+e_{v}+C_{G}\left[I_{W}\right]^{d}\left[\omega_{T}\right]^{d} \Omega-C_{T}\left[I_{W}\right]^{d}\right.\right.$ $\left.\left.\left[\omega_{G}\right]^{d} \Omega\right)\right]_{i}$ indicates components of each $\mathbb{R}^{4}$ control input, $N_{1}(t)$, $N_{2}(t) \in \mathbb{R}^{4 \times 8}$ are defined as

$$
N \triangleq\left[\begin{array}{l}
{\left[N_{1}\right]} \\
{\left[N_{2}\right]}
\end{array}\right]\left[\begin{array}{c}
\left(I_{8}-\hat{Q}_{w}^{+} \hat{Q}\right) \\
\left(I_{8}-\hat{Q}_{w}^{+} \hat{Q}\right)
\end{array}\right]
$$

and $\bar{s}_{w}(\delta), \quad \bar{s}_{g}(\delta) \in \mathbb{R}^{8 \times 4}$ are defined as $\bar{s}_{w} \triangleq\left[\begin{array}{ll}s_{w} & 0_{4 \times 4}\end{array}\right]^{T}$ and $\bar{s}_{g} \triangleq\left[\begin{array}{ll}0_{4 \times 4} & s_{g}\end{array}\right]^{T}$, respectively.

After substituting Eq. (27) into Eq. (25) and using Eq. (30b), the closed-loop dynamics for $\bar{r}\left(\omega, e_{0}, e_{v}\right)$ are given by

$$
J \dot{r}=-\frac{1}{2} \dot{J} r+Y_{1} \tilde{\theta}_{1}+Y_{2} \tilde{\theta}_{2}-k r-e_{v}
$$

where the notation $\tilde{\theta}_{1}(t) \in \mathbb{R}^{10}$ is defined as

$$
\tilde{\theta}_{1}=\theta_{1}-\hat{\theta}_{1}
$$

Based on Eq. (36) and the subsequent stability analysis, the parameter estimates $\hat{\theta}_{1}(t)$ and $\hat{\theta}_{2}(t)$ are designed as

$$
\dot{\hat{\theta}}_{1}=\operatorname{proj}\left(\Gamma_{1} Y_{1}^{T} r\right) \quad \dot{\hat{\theta}}_{2}=\operatorname{proj}\left(\Gamma_{2} Y_{2}^{T} r\right)
$$

where $\Gamma_{1} \in \mathbb{R}^{10 \times 10}$ and $\Gamma_{2} \in \mathbb{R}^{7 \times 7}$ are constant, positive-definite, diagonal adaptation gain matrices, and $\operatorname{proj}(\cdot)$ denotes a projection algorithm used to guarantee that the $i$ th element of $\hat{\theta}_{1}(t)$ and $\hat{\theta}_{2}(t)$ can be bounded as

$$
\underline{\theta}_{1 i} \leq \hat{\theta}_{1 i} \leq \bar{\theta}_{1 i} \quad \underline{\theta}_{2 i} \leq \hat{\theta}_{2 i} \leq \bar{\theta}_{2 i}
$$

where $\underline{\theta}_{1 i}, \bar{\theta}_{1 i} \in \mathbb{R}$ and $\underline{\theta}_{2 i}, \bar{\theta}_{2 i} \in \mathbb{R}$ are known, constant lower and upper bounds for each element of $\hat{\theta}_{1}(t)$ and $\hat{\theta}_{2}(t)$, respectively.

\section{B. Momentum Tracking Control Development}

The open-loop dynamics for the flywheel angular momentum tracking error $\mu(t)$ can be obtained by taking the time derivative of Eq. (17) as

$$
\dot{\mu}=-I_{W} \dot{\Omega}
$$

Multiplying Eq. (40) by the known positive-definite symmetric matrix $I_{W}^{-1}$ and substituting Eq. (34) into the resulting expansion for $\dot{\Omega}(t)$ yields

$$
I_{W}^{-1} \dot{\mu}=-R_{1}-N_{1} \bar{s}_{w} k_{w} g
$$

Based on the structure of Eq. (41), the null-space control $g(t)$ is designed to satisfy

$$
k_{w} N_{1} \bar{s}_{w} g=-R_{1}+k_{m} \mu
$$

where $k_{m} \in \mathbb{R}$ is a positive constant control gain. The minimum norm solution of Eq. (42) is 


$$
g=\left[k_{w} N_{1} \bar{s}_{w}\right]^{-1}\left(-R_{1}+k_{m} \mu\right)
$$

The result in Eq. (43) indicates that simultaneous attitude and momentum tracking is possible when $k_{w} N_{1}(\delta, t) \bar{s}_{w}(\delta)$ is invertible. After substituting Eq. (43) into Eq. (41), the closed-loop error system is

$$
I_{W}^{-1} \dot{\mu}=-k_{m} \mu
$$

Remark 1. The bracketed term in Eq. (43) is invertible, and simultaneous attitude and momentum tracking is possible when $N_{1}(\delta, t) \bar{s}_{w}(\delta)=\left(I_{8}-\hat{Q}_{w}^{+}(\delta, t) \hat{Q}(t)\right)_{4 \times 8} \bar{s}_{w}(\delta)_{8 \times 4} \neq 0_{4 \times 4}$. Because the projection matrix $\left(I_{8}-\hat{Q}_{w}^{+}(\delta, t) \hat{Q}(t)\right)$ is idempotent, $N_{1}(\delta, t) \neq$ $0_{4 \times 8}$ and simultaneous attitude and momentum tracking can be achieved provided

$$
\bar{s}_{w} \neq 0 \quad \text { and } \quad \bar{s}_{w} \notin \mathcal{N}\left(N_{1}\right)
$$

where $\mathcal{N}\left(N_{1}(\delta, t)\right)$ denotes the null space of the matrix $N_{1}(\delta, t)$. Hence, $\bar{s}_{w}(\delta) \notin \mathcal{N}\left(N_{1}\right)$ except for $\bar{s}_{w}(\delta)=0_{8 \times 4}$, which means the CMG's Jacobian itself is singular, but $\bar{s}_{w}(\delta)=0_{8 \times 4}$ does not occur for the CMG's Jacobian since $\varepsilon$ prevents the denominator of $s_{w}$ from being zero. Accordingly, the minimum norm solution of Eq. (42) exists, and simultaneous attitude and momentum tracking can be achieved.

\section{Stability Analysis}

Theorem 1: The weighted control input (27) along with the adaptive update laws given in Eq. (38) ensures global asymptotic attitude tracking such that

$$
\left\|e_{v}(t)\right\| \rightarrow 0 \quad \text { as } t \rightarrow \infty
$$

along with exponential internal momentum tracking in the sense that

$$
\|\mu(t)\| \leq \mu(0) \exp \left(-I_{W} k_{m} t\right)
$$

Proof: The exponential internal momentum tracking result is evident from Eq. (44).

To prove the attitude tracking result, let $\mathcal{D} \subset \mathbb{R}^{8}$ be a domain containing $\rho\left(e_{v}, e_{0}, r, P\right)=0$, where $\rho\left(e_{v}, e_{0}, r, P\right) \in \mathbb{R}^{8}$ is defined as

$$
\rho(t) \triangleq\left[e_{v}^{T}(t) \quad e_{0}(t) \quad r^{T}\left(e_{v}, e_{0}, \omega\right) \quad P\left(\tilde{\theta}_{1}(t), \tilde{\theta}_{2}(t)\right)\right]^{T}
$$

and the auxiliary function $P\left(\tilde{\theta}_{1}(t), \tilde{\theta}_{2}(t)\right) \in \mathbb{R}$ is defined as

$$
P \triangleq \frac{1}{2} \tilde{\theta}_{1}^{T} \Gamma_{1}^{-1} \tilde{\theta}_{1}+\frac{1}{2} \tilde{\theta}_{2}^{T} \Gamma_{2}^{-1} \tilde{\theta}_{2}
$$

and let $V(\rho, \delta): \mathcal{D} \times[0, \infty) \rightarrow \mathbb{R}$ be a continuously differentiable, positive-definite function defined as

$$
V \triangleq e_{v}^{T} e_{v}+\left(1-e_{0}\right)^{2}+\frac{1}{2} r^{T} J r+P
$$

After using Eqs. (13), (26), (6), (37), and (47), the time derivative of $V(\rho, \delta)$ can be expressed as

$$
\begin{aligned}
\dot{V} & =e_{v}^{T}\left(e_{v}^{\times}+e_{0} I\right) \tilde{\omega}+\left(1-e_{0}\right) e_{v}^{T} \tilde{\omega}+r^{T}\left(Y_{1} \tilde{\theta}_{1}+Y_{2} \tilde{\theta}_{2}-k r-e_{v}\right) \\
& -\tilde{\theta}_{1}^{T} \Gamma_{1}^{-1} \dot{\hat{\theta}}_{1}-\tilde{\theta}_{2}^{T} \Gamma_{2}^{-1} \dot{\hat{\theta}}_{2}
\end{aligned}
$$

By using Eqs. (19) and (38), and exploiting the fact that $e_{v}^{T}(t) e_{v}^{\times}(t) \tilde{\omega}(t)=0$, the expression in Eq. (49) can be upper bounded as

$$
\dot{V} \leq-\lambda\|z\|^{2}
$$

where $\lambda=\lambda_{\min }\{\alpha, k\} \in \mathbb{R}$, and $z\left(e_{v}, r\right) \in \mathbb{R}^{6}$ is defined as

$$
z \triangleq\left[e_{v}^{T}(t) \quad r^{T}\left(e_{v}, e_{0}, \omega\right)\right]^{T}
$$

From Eqs. $(\underline{48})$ and $(\underline{50}), r\left(e_{v}, e_{0}, \omega\right), e_{v}(t), e_{0}(t), \tilde{\theta}_{1}(t), \tilde{\theta}_{2}(t) \in \mathcal{L}_{\infty}$.
The facts that $r\left(e_{v}, e_{0}, \omega\right), e_{v}(t), e_{0}(t) \in \mathcal{L}_{\infty}$ indicate that $\omega(t)$, $\tilde{\omega}(t) \in \mathcal{L}_{\infty}$ from Eq. (15). The open-loop quaternion tracking error in Eq. (13) shows that $\dot{e}_{v}(t), e_{v}(t), \dot{e}_{0}(t) \in \mathcal{L}_{\infty}$ since $e_{v}(t), e_{0}(t)$, $\tilde{\omega}(t) \in \overline{\mathcal{L}_{\infty}}$. From Eq. (46), $\mu(t) \in \mathcal{L}_{\infty}$, and Eqs. (16) and (17) indicate that $\Omega(t) \in \mathcal{L}_{\infty}$. In Eq. (6), conservation of angular momentum, $\omega(t)$, and $\Omega(t) \in \mathcal{L}_{\infty}$ show that $\dot{\delta}(t) \in \mathcal{L}_{\infty}$. From Eq. (39), $\hat{\theta}_{1}(t), \hat{\theta}_{2}(t) \in \mathcal{L}_{\infty}$. Since $e_{v}(t), e_{0}(t), r\left(e_{v}, e_{0}, \omega\right), \omega(t)$, $\Omega(t) \in \mathcal{L}_{\infty}$ Eq. (34) can be used to show that $R_{1}(t) \in \mathcal{L}_{\infty}$. The inequality (39) can be used to prove that $R_{1}(t) \in \mathcal{L}_{\infty}$. With $R_{1}(t)$, $N_{1}(t), \mu(t) \in \mathcal{L}_{\infty}$, Eq. (43) indicates that $g(t) \in \mathcal{L}_{\infty}$. The fact that $r\left(e_{v}, e_{0}, \omega\right), e_{v}(t), \dot{\delta}(t), \tilde{\theta}_{1}(t), \tilde{\theta}_{2}(t) \in \mathcal{L}_{\infty}$ indicates that $\dot{r}\left(e_{v}, e_{0}, r, \dot{\delta}, \tilde{\theta}_{1}, \tilde{\theta}_{2}\right) \in \mathcal{L}_{\infty}$ from Eq. (자). Hence, Eqs. (27), (31), and (39), and the facts that $z\left(e_{v}, r\right), \Omega(t) \in \mathcal{L}_{\infty}$, can be used to prove that the control input $\dot{\eta}(t) \in \mathcal{L}_{\infty}$. Based on the aforementioned boundedness arguments, all remaining signals are bounded during closed-loop operation. Since $e_{v}(t), \quad r\left(e_{v}, e_{0}, \omega\right), \dot{e}_{v}(t)$, $\dot{r}\left(e_{v}, e_{0}, r, \dot{\delta}, \tilde{\theta}_{1}, \tilde{\theta}_{2}\right) \in \mathcal{L}_{\infty}, e_{v}(t)$ and $r\left(e_{v}, e_{0}, \omega\right)$ are uniformly continuous. Since $e_{v}(t)$ and $r\left(e_{v}, e_{0}, \omega\right)$ are uniformly continuous, and $e_{v}(t), r\left(e_{v}, e_{0}, \omega\right) \in \mathcal{L}_{\infty} \cap \mathcal{L}_{2}$, Barbalat's Lemma can be used to prove $r\left(e_{v}, e_{0}, \omega\right) e_{v}(t) \rightarrow 0$ as $t \rightarrow \infty$.

\section{Numerical Examples}

\section{A. Simulation Setup}

Numeric simulations illustrate the performance of the developed controller. The satellite parameters are based on a model of a prototype picosatellite and are given in Table 1 . A gimbal rate limit was included in the model as

$$
\operatorname{sat}\left(\dot{\delta}_{i}\right)=\left\{\begin{array}{cl}
\dot{\delta}_{i}, & \text { for }\left|\dot{\delta}_{i}\right| \leq 25(\mathrm{rad} / \mathrm{sec}) \\
25 \operatorname{sgn}\left(\dot{\delta}_{i}\right), & \text { for }\left|\dot{\delta}_{i}\right|>25(\mathrm{rad} / \mathrm{sec})
\end{array} \quad \forall i=1,2,3,4\right.
$$

where $\operatorname{sgn}(\cdot)$ denotes the standard signum function. The desired angular velocity trajectories $\omega_{d}(t)$ are $\omega_{d}(t)=[0.004 \sin (2 \pi t /$ $2000) 00](\mathrm{rad} / \mathrm{s})$, and the desired flywheel speed for each wheel is $\Omega_{d}=200 \mathrm{rad} / \mathrm{s}(\approx 2000 \mathrm{rpm})$. The initial conditions are given in Table 2 .

\section{B. Simulation Results}

The simulation results are developed for two cases. Case 1 is included to illustrate how the controller responds when the momentum tracking has a long transient. For this case, the VSCMG has to operate in RW mode during the transient, resulting in gimbal rate saturation. The results are given in Figs. 2-9. Case 2 is included to illustrate a more favorable condition (which can be achieved through control gains) where the momentum tracking error has a short transient response. Results in Figs. 10-13 illustrate that, for this case, gimbal rate saturation is avoided and the VSCMG operates in

Table 1 Physical parameters for the VSCMG simulation

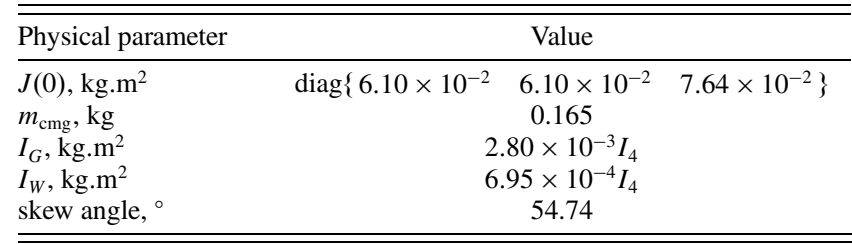

Table 2 Initial parameters for the VSCMG simulation

\begin{tabular}{lcc}
\hline \hline Initial parameter & Value \\
\hline$q(0)$ & {$\left[\begin{array}{llll}0.1 & 0.3 & 0.8 & 0.4\end{array}\right]$} \\
$\omega(0), \mathrm{rad} / \mathrm{s}$ & 0 & \\
$\hat{\theta}_{1}(0), \hat{\theta}_{2}(0)$ & 0 & \\
$\delta(0), \mathrm{rad}$ & {$\left[\begin{array}{llll}0.5498 & 0.2333 & 0.5498 & 0.2333\end{array}\right]$} \\
$\Omega(0), \mathrm{rad} / \mathrm{s}$ & 0 & \\
\hline \hline
\end{tabular}



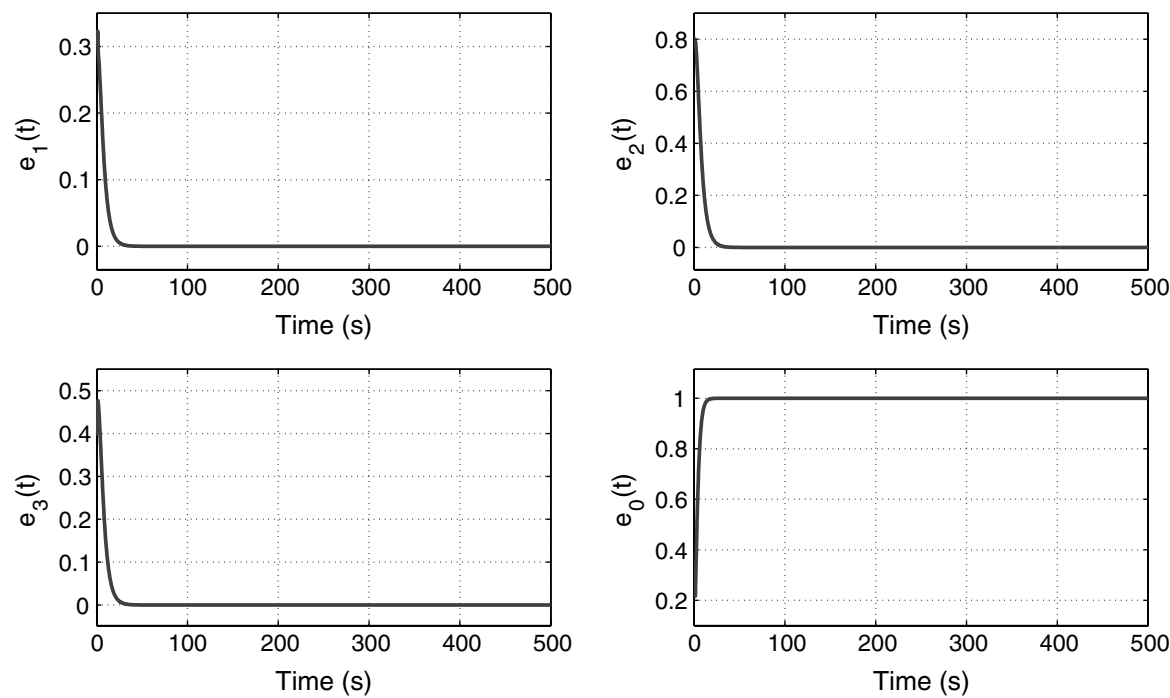

Fig. 2 Quaternion tracking error $e(t)$ for case 1.
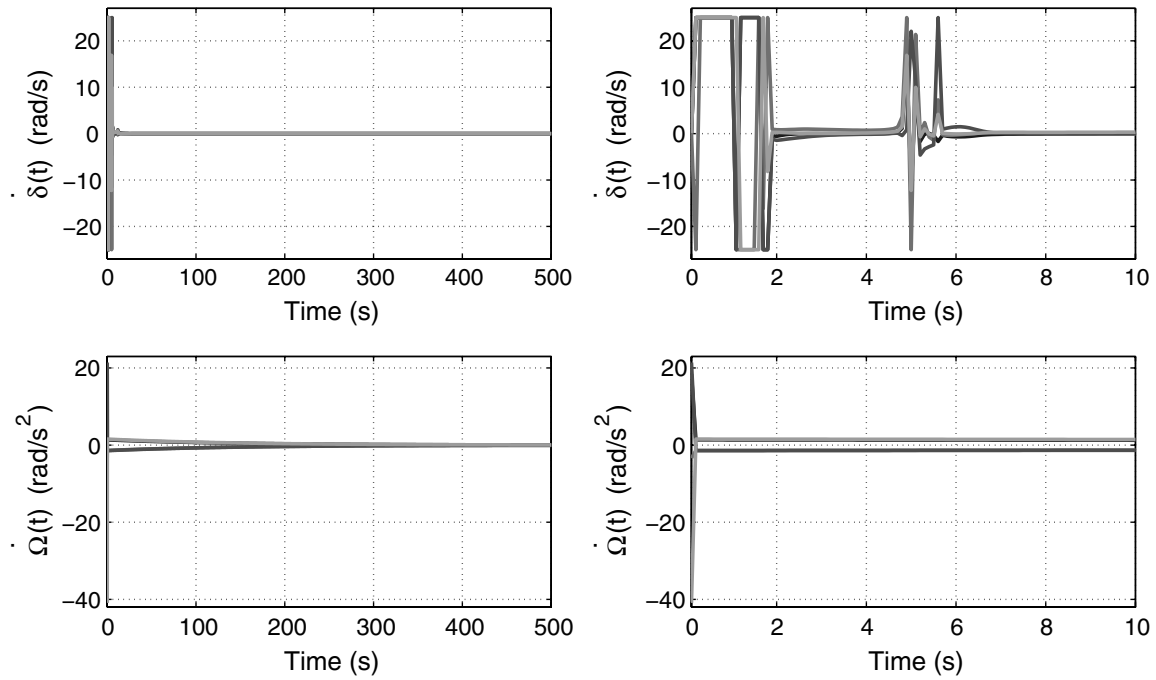

Fig. 3 Control input gimbal rates $\dot{\delta}(t)$ (top) and wheel accelerations $\dot{\Omega}(t)$ (bottom) for case 1 .

RW mode less than for case 1 . The transient response of internal momentum tracking is adjustable corresponding to tuning of control gain $k_{m}$ as seen in Eq. (43). In Fig. 4, the actual flywheel speed of case 1 approaches the desired flywheel speed as a steady-state response at over a $500-\mathrm{s}$ simulation period $(\approx 600 \mathrm{~s})$, which is regarded as a longer transient case. In case 2 , the actual flywheel speed achieves shorter transient response than case 1 as seen by Fig. 11 (i.e., the settling time is 200 s.). Figure 2 shows the quaternion tracking error results to achieve the control objective denoted in Eq. (14) during a 500-s simulation period. Figure 3 shows the control input gimbal rates $\dot{\delta}(t)$ and wheel accelerations $\dot{\Omega}(t)$. While the control inputs achieve attitude stabilization, the wheel acceleration control input $\dot{\Omega}(t)$ contributes to the internal momentum management by regulating the wheel speed after starting from rest. Figure 4 indicates the initial startup of flywheels from rest. To compensate for the lack of torque generated by the flywheel due to the slow momentum tracking, the gimbal rates in Fig. 3 are shown to generate more torques (including torque saturation) during the transient response. The increased transient response of the gimbal rate yields increased singularities in the CMG Jacobian, but these singularities are effectively avoided as shown in Figs. $\underline{5}$ and 6 . As a result of approaching singularities, the flywheels are required to operate in RW mode as shown in Fig. 4. The null-motion weight $S$ in Eq. (33), depicted in Fig. 5, has an increased transient in this case because $S$ inhibits the momentum tracking when the CMG Jacobian approaches a singularity so that the flywheel can work in RW mode. Although $S$ cannot distinguish between different singularities, the weight matrix can adjust the intervention of the flywheel when approaching a singularity. Hence, the composite weighted steering law in Eq. (27) can cope with an elliptic singularity while maintaining precision attitude control because the steering law generates the required torque in RW mode to pass through or escape an internal singularity differently from the singularity escape methods for CMGs that require added torque $[8,10-12,14,36]$. This observation indicates a benefit that results from the extra controllable degree of freedom of the VSCMG. Specifically, $s_{w}$ in Eq. (33), depicted in Fig. 5, allows momentum tracking when in normal operation and restricts the momentum tracking when approaching a singularity. Thus, in most regions, gimbal reconfiguration is responsible for singularity avoidance by exploiting gimbal rate null solution. The null-motion weight $S$ serves as a switch that acts as an alternative to cope with both elliptic and hyperbolic singularities while achieving internal momentum management. This benefit provides an avenue to effectively acquire the initial startup without a separate feedback loop. The time variation of the adaptive parameter estimates is shown in Figs. 7-9. Figure 7 shows the elements of the adaptive parameter vectors $\hat{\theta}_{1}(t)$ and $\hat{\theta}_{2}(t)$. Figures $\underline{8}$ and $\underline{9}$, divided into multiple windows for clarity, highlight the adaptive parameter estimates of 


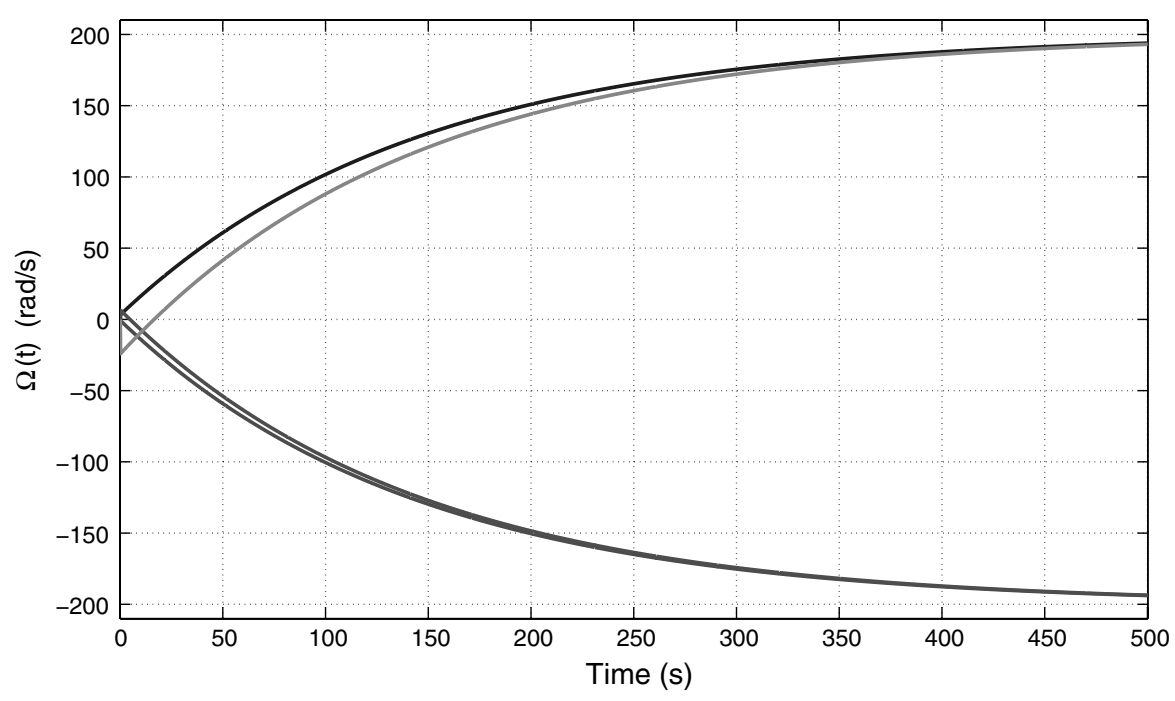

Fig. 4 Flywheel speed $\Omega(t)$ induced from internal momentum management for case 1.
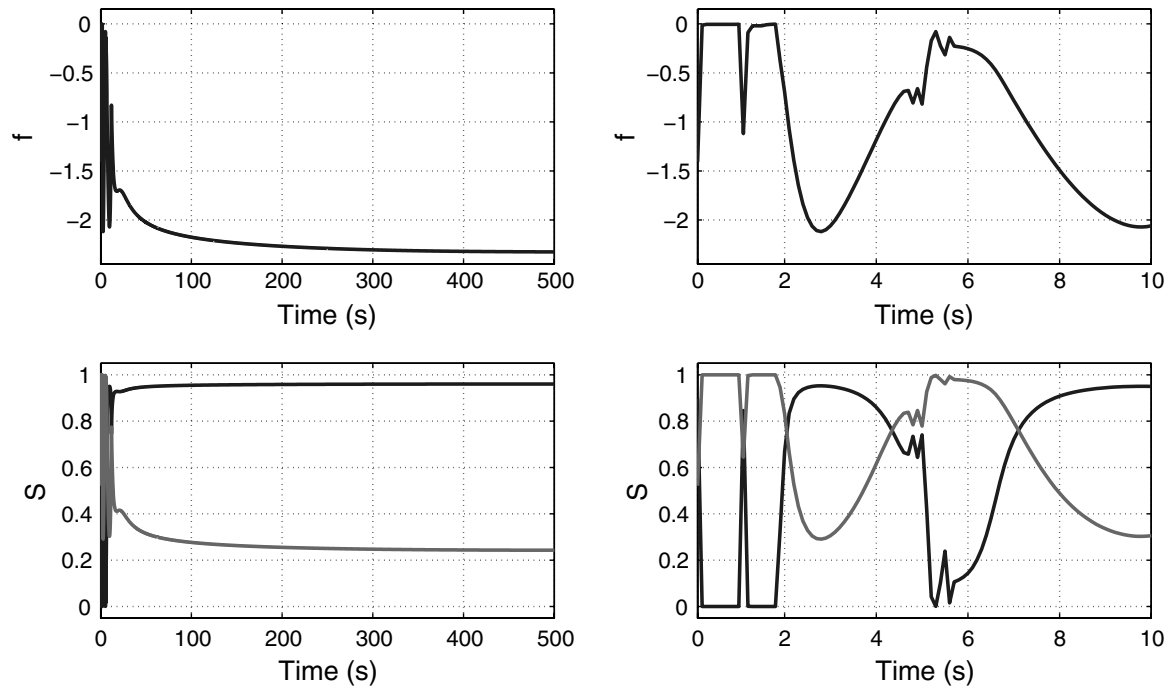

Fig. 5 Singularity measure function $f$ (top) and null-motion weight $S$ (bottom) for case 1.
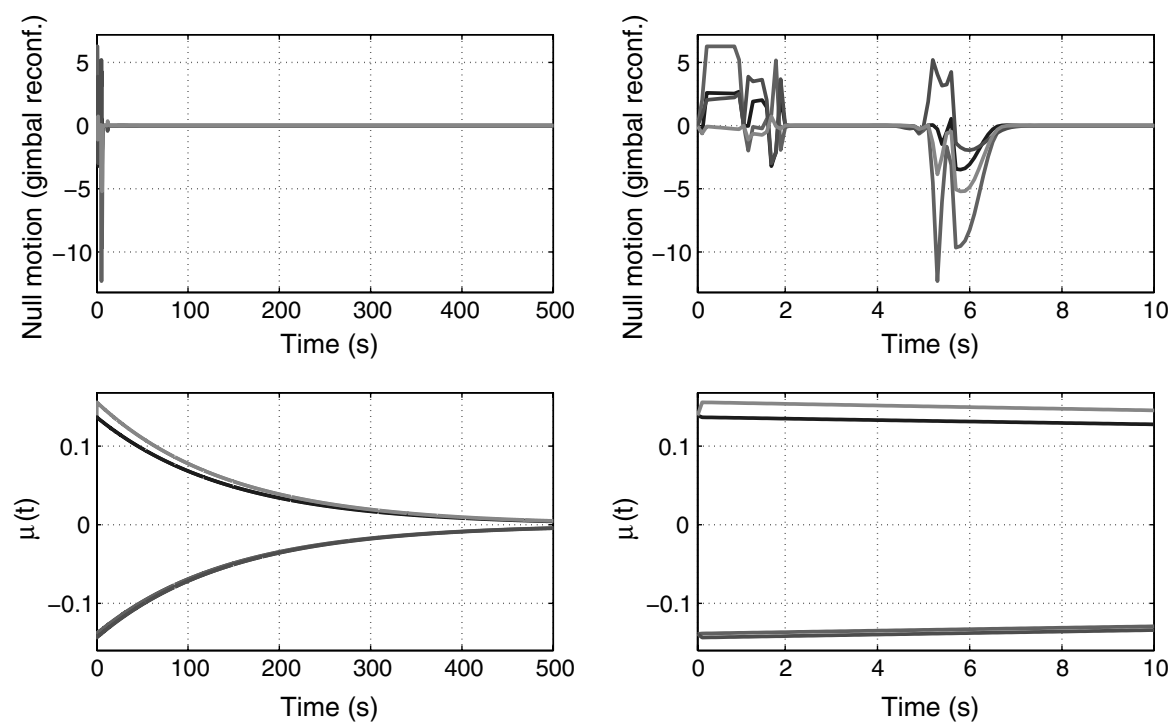

Fig. 6 Null motion: gimbal reconfiguration (top) and momentum tracking error (bottom) for case 1. 

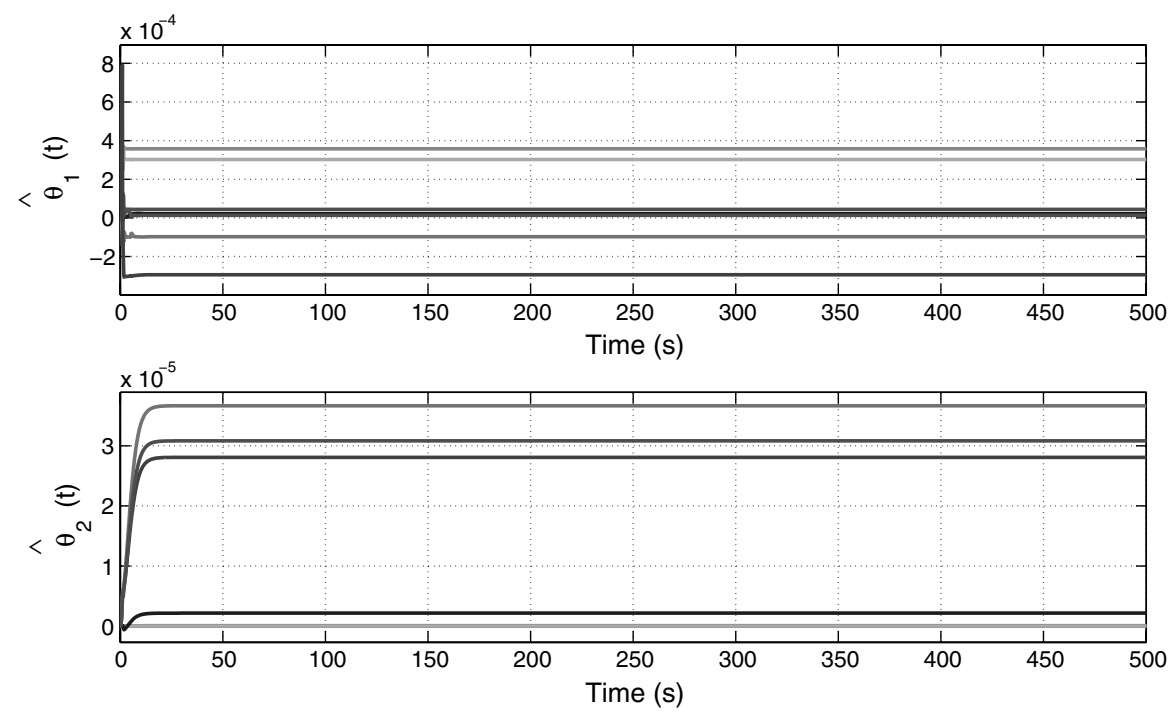

Fig. 7 Adaptive parameter estimates $\hat{\theta}_{1}(t)$ and $\hat{\theta}_{2}(t)$ for case 1.
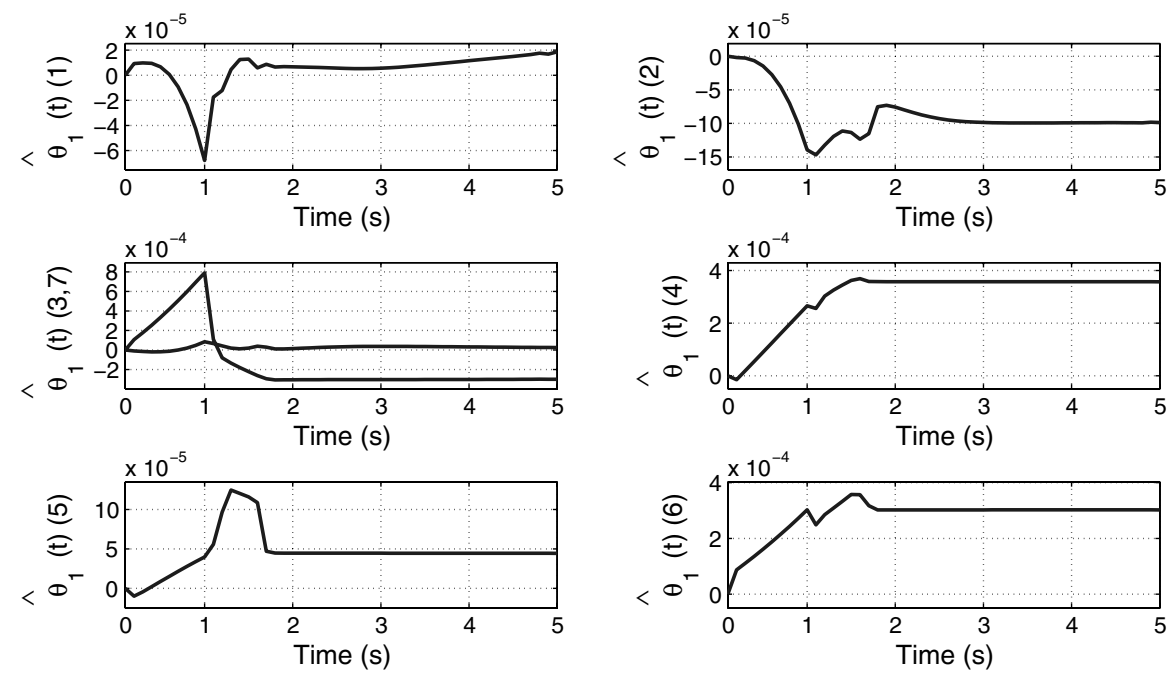

Fig. 8 Transient response of adaptive parameter estimate $\hat{\theta}_{1}(t)$ for case 1. The notation $\hat{\theta}_{1}(t)(x)$ denotes the $x$ element of $\hat{\theta}_{1}(t)$.
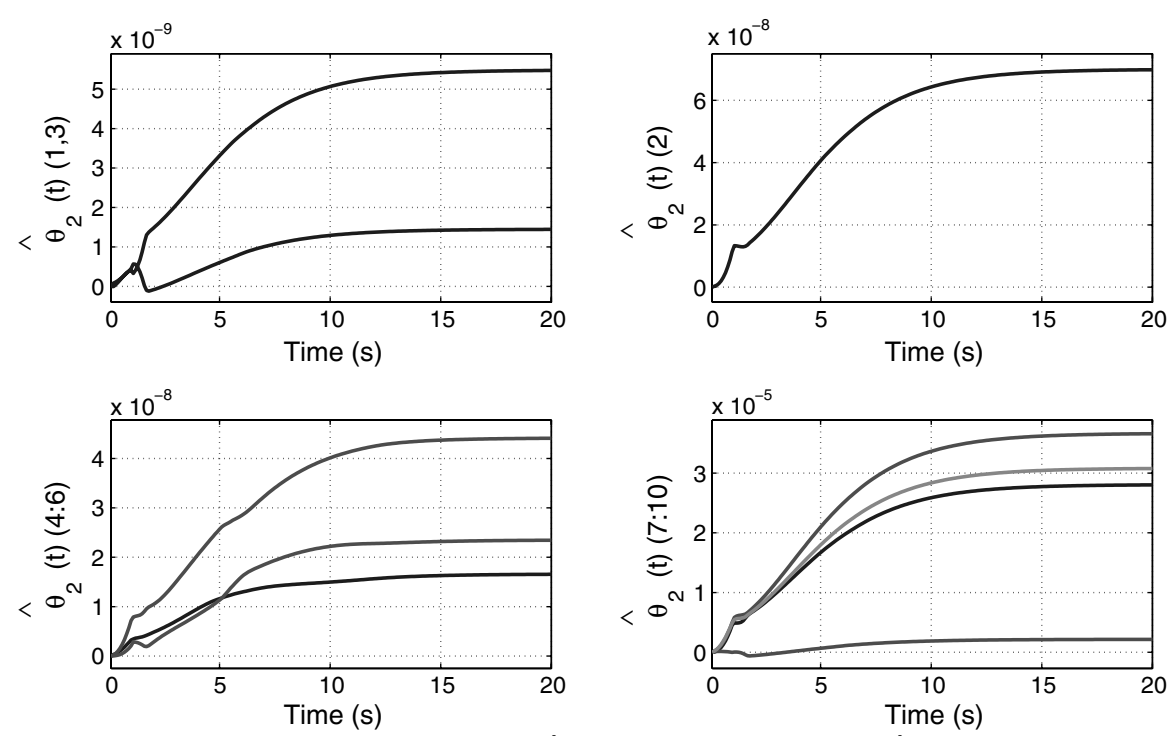

Fig. 9 Transient response of adaptive parameter estimate $\hat{\theta}_{2}(t)$ for case 1. The notation $\hat{\theta}_{2}(t)(x)$ denotes the $x$ element of $\hat{\theta}_{2}(t)$. 

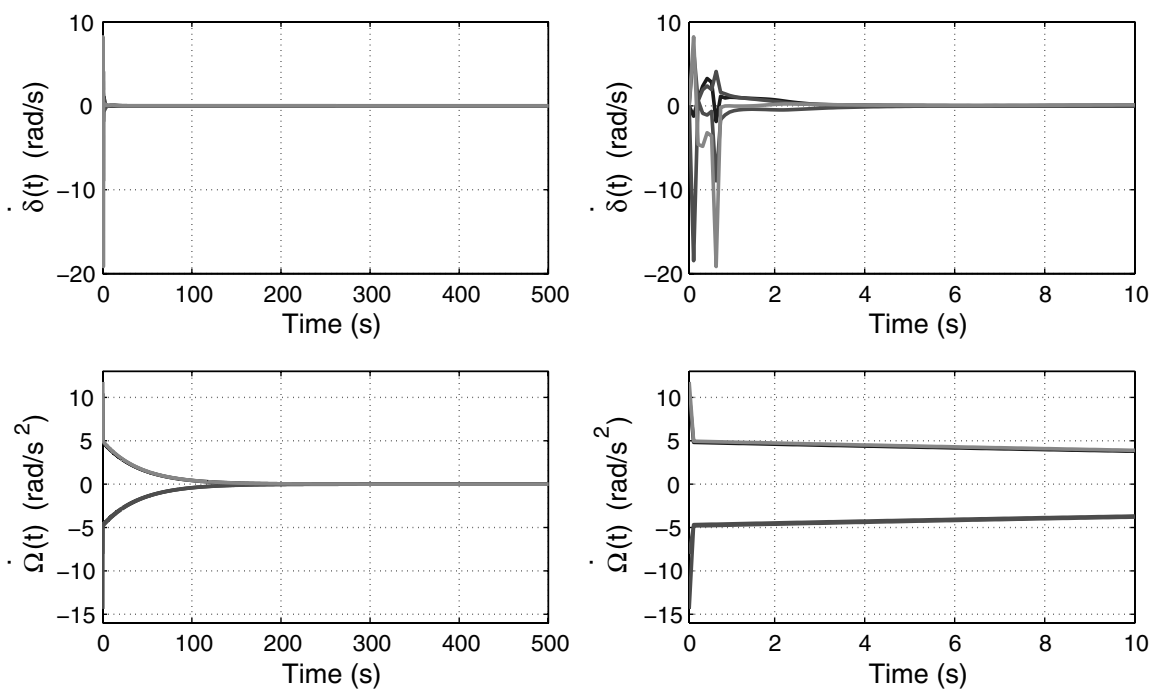

Fig. 10 Control input gimbal rates $\dot{\delta}(t)$ (top) and wheel accelerations $\dot{\Omega}(t)$ (bottom) for case 2.

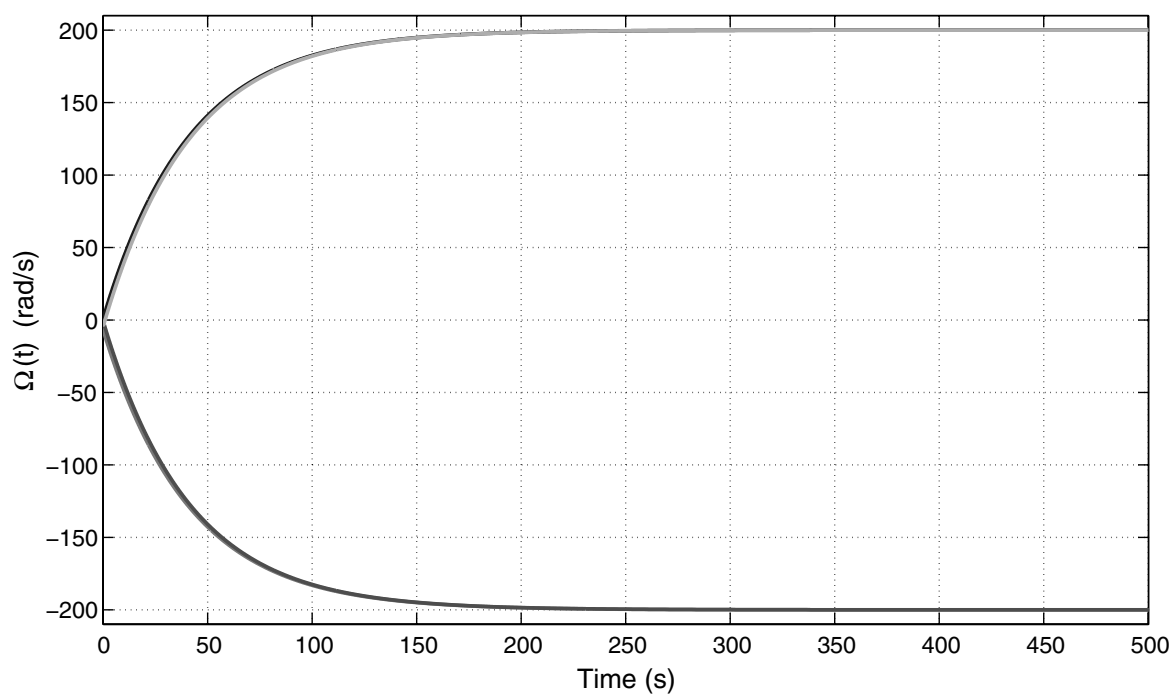

Fig. 11 Flywheel speed $\Omega(t)$ induced from internal momentum management for case 2.
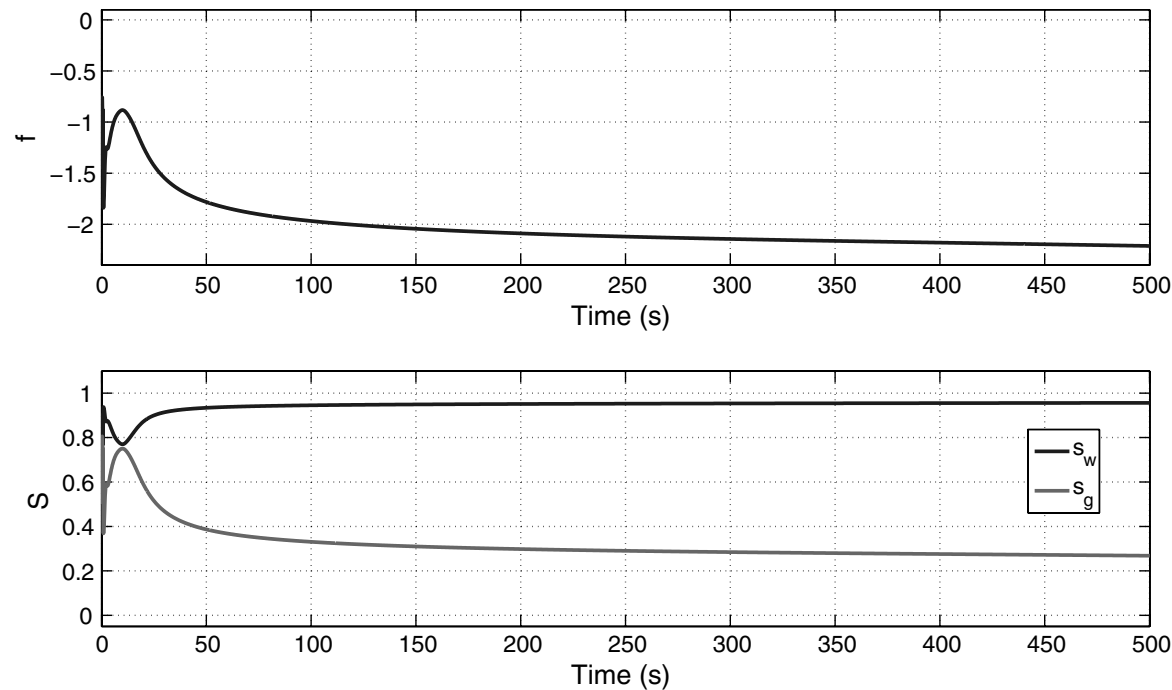

Fig. 12 Singularity measure function $f$ (top) and null-motion weight $S$ (bottom) for case 2. 

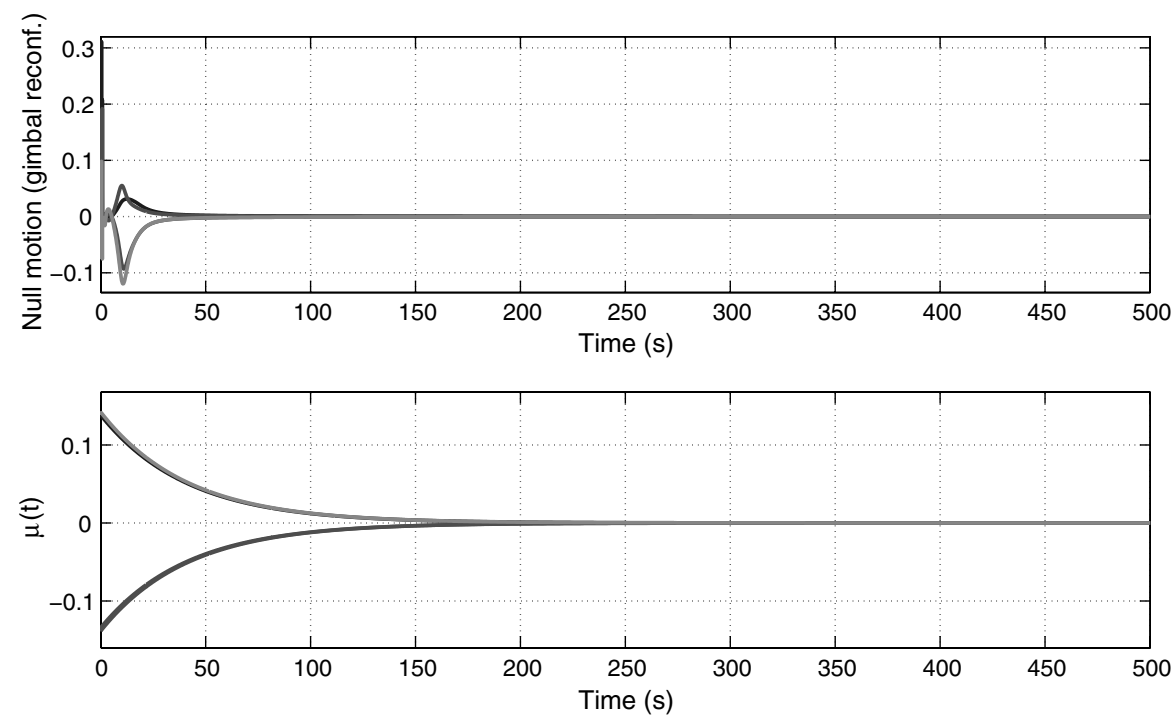

Fig. 13 Null motion: gimbal reconfiguration (top) and momentum tracking error (bottom) for case 2.

$\hat{\theta}_{1}(t), \hat{\theta}_{2}(t)$, respectively. The adaptation mechanism adjusts the uncertain parameter estimate so that attitude tracking can be asymptotically achieved. Moreover, the initial values of the uncertain parameters are chosen to be zero, indicating no a priori parameter knowledge. Even though some of the parameter estimates indicate the variation by small magnitudes, the equivalent control torques arising from the feedforward terms $\hat{\theta}_{1}(t), \hat{\theta}_{2}(t)$, resulting from Eqs. (7), (22), (23), (27), and (ㅍ), have similar magnitudes to that of the feedback control terms.

Case 2 is developed for the closed-loop operations in fast momentum tracking and the simulated results are provided in Figs. 10-13. Figure 10 shows the gimbal rate $\dot{\delta}(t)$ and the wheel acceleration $\dot{\Omega}(t)$ control inputs. Figure 11 shows that the flywheel speed tracks the desired wheel speed from rest. The momentum tracking gain $k_{m}$ denoted in Eq. (42) controls the momentum tracking speed. Because the flywheels rapidly arrive at the desired constant speed, fast momentum tracking allows the VSCMG steering law to operate longer in the CMG mode, which provides torque amplification and power savings. Figure 12 illustrates the singularity measure function $f$ and the null-motion weight $S$. Corresponding to the moderate variation of $f, s_{w}$ allows steady momentum tracking, and $s_{g}$ generates the proper gimbal reconfiguration as depicted in Fig. 13.

\section{Conclusions}

In the presence of satellite inertia uncertainty and actuator uncertainty, the developed controller is capable of achieving global asymptotic attitude tracking while simultaneously performing singularity avoidance and internal momentum management. The benefits such as singularity avoidance and internal momentum management emerge from the null solution of the control inputs. In particular, the internal momentum management allows the flywheel to start from rest and to reach the desired speed. To maximize operation in control moment gyroscope mode, the steering law exploits the singularity avoidance strategy resulting from the gradient method, and the null-motion weight adjusts the internal momentum tracking of the flywheels when approaching an internal singularity. The variable-speed control moment gyroscope (VSCMG)-actuated satellite can accomplish asymptotic attitude tracking and exponential internal momentum tracking while simultaneously achieving singularity avoidance. The adaptive controller-based VSCMG steering law also compensates for the effects of uncertain, time-varying satellite inertia properties. The difficulties arising from the uncertain satellite inertia are mitigated through an innovative development of the error system along with a Lyapunov-based adaptive law. The attitude tracking and momentum tracking results are proven via a Lyapunov stability analysis and demonstrated through numerical simulations.

\section{References}

[1] Chang, Y., Lee, B., and Kim, S., "Momentum Wheel Start-Up Method for HAUSAT-2 Ultra-Small Satellite," Aerospace Science and Technology, Vol. 10, No. 2, 2006, pp. 168-174. doi:10.1016/j.ast.2005.07.011

[2] Hamzah, N., and Hashida, Y., "TiungSAT-1: From Inception to Inauguration," TiungSAT-1 Momentum Wheel Commissioning, Astronautic Technology, 2001, pp. 73-92.

[3] Lafon, T., and Parisot, F., "The Jason-1 Satellite Design and Development Status," Proceeding of 12th AIAA/USU Conference on Small Satellite, AIAA Paper SSC98-V-6, 1998.

[4] Ford, K. A., and Hall, C. D., "Flexible Spacecraft Reorientations Using Gimbaled Momentum Wheels," Advances in the Astronautical Sciences, Vol. 97, No. 3, 1997, pp. 1895-1914.

[5] Kurokawa, H., "Survey of Theory and Steering Laws of Single-Gimbal Control Moment Gyros," Journal of Guidance, Control, and Dynamics, Vol. 30, No. 5, 2007, pp. 1331-1340. doi: $10.2514 / 1.27316$

[6] Lappas, V., Steyn, W., and Underwood, C., "Attitude Control for Small Satellites Using Control Moment Gyros," Acta Astronautica, Vol. 51, No. 1, 2002, pp. 101-111. doi:10.1016/S0094-5765(02)00089-9

[7] Margulies, G., and Aubrun, J. N., "Geometric Theory of Single-Gimbal Control Moment Gyro Systems," Journal of the Astronautical Sciences, Vol. 26, No. 2, 1978, pp. 159-191.

[8] Oh, H., and Vadali, S., "Feedback Control and Steering Laws for Spacecraft Using Single Gimbal Control Moment Gyro," Journal of the Astronautical Sciences, Vol. 39, No. 2, 1991, pp. 183-203.

[9] Wie, B., Bailey, D., and Heiberg, C., "Rapid Multitarget Acquisition and Pointing Control of Agile Spacecraft," Journal of Guidance, Control, and Dynamics, Vol. 25, No. 1, 2002, pp. 96-104. doi: $10.2514 / 2.4854$

[10] Leve, F., and Fitz-Coy, N., "Hybrid Steering Logic for Single-Gimbal Control Moment Gyroscopes," Journal of Guidance, Control, and Dynamics, Vol. 33, No. 4, July-Aug. 2010, pp. 1202-1212. doi: $10.2514 / 1.46853$

[11] Wie, B., Bailey, D., and Heiberg, C., "Singularity Robust Steering Logic for Redundant Single-Gimbal Control Moment Gyros," Journal of Guidance, Control, and Dynamics, Vol. 24, No. 5, 2001, pp. $865-872$. doi:10.2514/2.4799

[12] Ford, K. A., and Hall, C. D., "Singular Direction Avoidance Steering for Control-Moment Gyros," Journal of Guidance, Control, and Dynamics, Vol. 23, No. 4, 2000, pp. 648-656. doi: $10.2514 / 2.4610$

[13] Kurokawa, H., "A Geometry of Single Gimbal Control Moment Gyros-Singularity Problem and Steering Law," Mechanical Engineering Lab. TR 175, Tsukuba, Japan, Jan. 1998.

[14] Bedrossian, N., Paradiso, J., Bergmann, E., and Rowell, D., "Steering 
Law Designs for Redundant SGCMG Systems," Journal of Guidance, Control, and Dynamics, Vol. 13, No. 6, 1990, pp. 1083-1089. doi: $10.2514 / 3.20582$

[15] Busseuil, J., Llibre, M., and Roser, X., "High Precision Mini-CMG's and their Spacecraft Applications," AAS Guidance and Control Conference, Univelt, San Diego, CA, 1998, pp. 91-107.

[16] Hoelscher, B., and Vadali, S., "Optimal Open-Loop and Feedback Control Using Single Gimbal Control Moment Gyroscopes," Journal of the Astronautical Sciences, Vol. 42, No. 2, 1994, pp. 189-206.

[17] Konda, Y., Usuda, T., Sagami, T., Omagari, K., Kashiwa, M., and Matunaga, S., "Development of Attitude Determination and Control System for Pico-Satellite Cute-1.7 + APD," The 16th Workshop on JAXA Astrodynamics and Flight Mechanics, Kawaguchi Lab., Sagamihara, Japan, 2006, pp. 242-247.

[18] Krishnan, S., and Vadali, S., "An Inverse-Free Technique for Attitude Control of Spacecraft Using CMGs," Acta Astronautica, Vol. 39, No. 6, 1996, pp. 431-438. doi:10.1016/S0094-5765(96)00152-X

[19] Lappas, V., Underwood, C., and Steyn, W., "Experimental Testing of a CMG Cluster for Agile Microsatellites," Proceeding of the 54th International Astronautical Congress of the IAF, International Astronautical Federation, 2003, pp. 371-377.

[20] Leve, F., Tatsch, A., and Fitz-Coy, N., "Scalable Control Moment Gyro Design for Attitude Control of Micro-, Nano-, and Pico-Class Satellites," AAS Guidance and Control Conference, American Astronautical Society Paper AAS 07-041, Breckenridge, CO, 2007.

[21] Leve, F., Nagabhushan, V., and Fitz-Coy, N., "P-n-P Attitude Control System for Responsive Space Missions," Responsive Space Conference, Microcosm Paper RS7-2009-5001, April 2009.

[22] Leve, F., Boyarko, G., and Fitz-Coy, N., "Precise Torque Mapping of Pico-Satellite Single-Gimbal Control Moment Gyroscopes," AAS Guidance and Control Conference, American Astronautical Society Paper AAS 10-095, Breckenridge, CO, 2010.

[23] MacKunis, W., Dupree, K., Fitz-Coy, N., and Dixon, W. E., "Adaptive Satellite Attitude Control in the Presence of Inertia and CMG Gimbal Friction Uncertainties," Journal of the Astronautical Sciences, Vol. 56, No. 1, 2008, pp. 121-134.

[24] Bradford, A., Gomes, L., Sweeting, M., Yukse, G., Ozkaptan, C., and Orlu, U., "BILSAT-1: A Low-Cost, Agile, Earth Observation Microsatellite for Turkey," Acta Astronautica, Vol. 53, No. 4, 2003, pp. 761-769. doi:10.1016/S0094-5765(03)00125-5

[25] Montfort, E., Kessab, M., Rennie, M., McGuinness, D., Palomo, P.,
Zwartbol, T., and Oving, B., "Real-Time Test Bench with Control Moment Gyro in the Loop," Proceeding of the 6th International ESA Conference on Guidance, Navigation and Control Systems, European Space Agency Paper ESA sp-606, 2006.

[26] Nishida, J., and Tsubuku, Y., “Tokyo Tech's Technical Demonstration Satellite TSUBAME," Proceeding of the 21st Small Satellite Conference, Tokyo Inst. of Technology Paper SSC07-IX-2, 2007.

[27] Schaub, H., and Junkins, J., Analytical Mechanics of Space Systems, AIAA Education Series, AIAA, Reston, VA, 2003, pp. 158-159.

[28] Schaub, H., Vadali, S. R., and Junkins, J. L., "Feedback Control Law for Variable Speed Control Moment Gyros," Journal of the Astronautical Sciences, Vol. 46, No. 3, July 1998, pp. 307-328.

[29] Yoon, H., and Tsiotras, P., "Singularity Analysis of Variable-Speed Control Moment Gyros," Journal of Guidance, Control, and Dynamics, Vol. 27, No. 3, 2004, pp. 374-386. doi: $10.2514 / 1.2946$

[30] Schaub, H., and Junkins, J. L., "Singularity Avoidance Using Null Motion and Variable-Speed Control Moment Gyros," Journal of Guidance, Control, and Dynamics, Vol. 23, No. 1, 2000, pp. 11-16. doi: $10.2514 / 2.4514$

[31] Yoon, H., and Tsiotras, P., "Spacecraft Adaptive Attitude and Power Tracking with Variable Speed Control Moment Gyroscopes," Journal of Guidance, Control, and Dynamics, Vol. 25, No. 6, Nov.-Dec. 2002, pp. 1081-1090. doi: $10.2514 / 2.4987$

[32] Hughes, P., Spacecraft Attitude Dynamics, Wiley, New York, 1994, pp. 26-27.

[33] Dixon, W. E., Behal, A., Dawson, D. M., and Nagarkatti, S., Nonlinear Control of Engineering Systems: A Lyapunov-Based Approach, Birkhäuser Boston, Cambridge, MA, 2003, pp. 229-233.

[34] Richie, D. J., Tsiotras, P., and Fausz, J. L., "Simultaneous Attitude Control and Energy Storage Using VSCMGs: Theory and Simulation," Proceedings of American Control Conference, IEEE Publications, Piscataway, NJ, June 2001, pp. 3973-3979.

[35] Wie, B., "Singularity Analysis and Visualization for Single-Gimbal Control Moment Gyros Systems," Journal of Guidance, Control, and Dynamics, Vol. 27, No. 2, March-April 2004, pp. 271-282. doi: $10.2514 / 1.9167$

[36] Wie, B., "New Singularity Escape and Avoidance Steering Logic for Control Moment Gyro Systems," Proceeding of AIAA Guidance, Navigation and Control Conference, Paper 2003-5659, AIAA, Reston, VA, Aug. 2003, pp. 2191-2201. 